\title{
Exploring the Relationship Between International Classification of Functioning, Disability, and Health Items Linked to Clinical Assessments in Children With Cerebral Palsy
}

\author{
Sang-Duk Park ${ }^{1}$, MS, Sook-Hee $\mathrm{Yi}^{2}$, MD, Jeong-Soo Kim¹,3, PhD \\ ${ }^{1}$ Department of Physical Therapy, Seoul Rehabilitation Hospital, '2Department of Rehabilitation Medicine, Seoul Rehabilitation Hospital, \\ Seoul, ${ }^{3}$ Department of Physical Therapy, College of Health Science, Yonsei University, Wonju, Korea
}

\author{
Article Info \\ Received October 1, 2021 \\ Revised October 12, 2021 \\ Accepted October 19, 2021 \\ Corresponding Author \\ Jeong-Soo Kim \\ E-mail: suah7475@hanmail.net \\ https://orcid.org/0000-0001-9994-9360
}

\section{Key Words}

Cerebral palsy

GMFM-66

ICF-CY

Outcome measures

PEDI

\begin{abstract}
Background: The International Classification of Functioning, Disability, and Health-Child and Youth version (ICF-CY) is designed to record the characteristics of developing children and examine the influence of a child's environment on their health.
\end{abstract}

Objects: This study was designed to determine the relationship between the clinically extracted ICF-CY items and The Pediatric Evaluation of Disability Inventory (PEDI) and Gross Motor Function Measure (GMFM) items.

Methods: Thirty patients (17 males and 13 females) who were hospitalized in a pediatric and youth patient unit of a rehabilitation hospital were included in the study. Four health professionals (two physical therapists and two occupational therapists) working independently linked the PEDI and GMFM-66 items to the activity and participation domains of the ICF-CY.

Results: There were strong negative correlations between the ICF-CY subdomains and the PEDI subdomains $(r=0.76-0.95 ; p<0.05)$. There were positive strong correlations between the ICF-CY subdomains and the GMFM-66 ( $r=0.76-0.95 ; p<0.05)$.

Conclusion: The extracted ICF codes were a valid tool for evaluating the mobility and selfcare conditions of cerebral palsy in the pediatric rehabilitation area.

\section{INTRODUCTION}

The World Health Organization (WHO) announced the International Classification of Functioning, Disability, and Health (ICF) by presenting a common language and framework that referred to health and health-related states in terms of body function and body structure, activity, and participation [1]. The International Classification of Functioning, Disability, and Health-Child and Youth version (ICF-CY) is an extended version of the ICF and special attention is given to the aspects of behavior, learning, and development of children and youth [2]. The ICF-CY can be used as a framework for human functioning and disability, which are considered a result of the dynamic interaction between health conditions and contextual factors.

The ICF-CY framework can be used as a common reference to compare the content of clinical outcome measures [3]. Mapping clinical outcome measurements to the ICF-CY coding system can provide information on health areas, disabilities, and functions applied by these measurements. In the field of rehabilitation for children with disabilities, comprehensive communication between team members and the use of common terminology according to the personal conditions, health condition and environmental factors are essential [4].

The Pediatric Evaluation of Disability Inventory (PEDI) is a widely used clinical assessment that samples the key functional capabilities and performance of children between the ages of 6 months and 7.5 years [5] The PEDI was designed to assess what the child actually does in the activity rather than describe his or her impairments (body functions and body systems). The functional skills section of the PEDI provides summary scores that reflect the child's current repertoire of daily life skills in each of three domains (self-care, mobility, and social function), while the caregiver assistance section provides a summary scores of the extent to which the child's total perfor- 
mance of complex daily life tasks such as dressing or moving around is supported by help from a caregiver. The Gross Motor Function Measure (GMFM) was used to assess the subjects' gross motor function. This measure comprises five dimensions of (A) lying and rolling, (B) sitting, (C) crawling and kneeling, (D) standing, and (E) walking, running, and jumping [6].

The ICF-CY is actually used in clinical practice, but there is a lack of research on whether it fully reflects the evaluation tools used by clinical experts in each specialized field. Therefore, it is importance to find out whether ICF-CY items reflect the contents of representative functional evaluation tools in physical and occupational treatment areas such as PEDI and GMFM.

Children with cerebral palsy (CP) are certainly limited in their daily activities and capacity to participate [7]. Their condition not only restricts their social participation but also their overall quality of life [8]. The activity and participation of affected children vary depending upon the severity of their condition, age, self-management, and movement and function in society [9]. Thus, the purpose of treatment for a child with $\mathrm{CP}$ is to facilitate motor function whereby he/she can engage in activities and participate in the community.

The ICF-CY sets are essential in providing a practical way to address a specific patient population in daily practice. The ICF-CY and PEDI are grounded in a similar conceptual model. However, there is a lack of research on how the assessment tools used by clinical professionals in each specialty compare to the ICF-CY framework. The aim of this study was to develop standardized ICF-CY sets linked to specific chapters of the PEDI and Gross Motor Function Measure (GMFM).

\section{MATERIALS AND METHODS}

\section{Participants}

Thirty patients (17 males and 13 females) who were hospitalized in a pediatric and youth patient unit of rehabilitation hospital were included in our study. The inclusion criteria were a diagnosis of uni- or bilateral, dyskinetic $\mathrm{CP}$, those who were classified at Gross Motor Function Classification System (GMFCS) levels I-IV, and patients under 18 years of age. The study was approved by the Seoul Rehabilitation Hospital Institutional Review Board (No. SRH2013R-01). Table 1 lists the general characteristics of the participants.

\section{Procedure}

Four health professionals (two physical therapists and two occupational therapists) working independently linked the PEDI and GMFM-66 items to the activity and participation domains of the ICF-CY using the same coding process described in a pre-meeting and then resolved disagreements by consensus. The two physical therapists and two occupational therapists had more than five years of clinical experience and wide knowledge and training in the ICF-CY model. A total of five consensus meetings were held to reach an agreement about the connections between the ICF-CY codes and items. The linking process followed the linking rules proposed by Cieza et al. [10], which involved linking the meaningful concept associated with each of the PEDI and GMFM-66 items to the ICF-CY codes by a manifest and deductive content analysis procedure. A meaningful concept was described as a set of words with one key meaning identified. If an item contained more than one concept, each concept was linked separately.

The PEDI and GMFM-66 were performed by two researchers before and after a 12-week treatment session. Treatment was carried out in everyday situations three times a week at the rehabilitation hospital. The authors did not participate in treating the children.

The PEDI is a standardized tool using parental reports through structured interviews and consists of the Functional Skills Scale (FSS), and the Caregiver Assistance Scale (CAS), both consisting of three domains of self-care, mobility, and social function. For the present study, the self-care and mobil-

Table 1. Descriptive data of all subjects $(N=30)$

\begin{tabular}{llc}
\hline \multicolumn{1}{c}{ Variables } & Categories & $\mathrm{n}$ \\
\hline Sex & Male & 17 \\
Age lyr) & Female & 13 \\
Classification of CP & Spastic bilateral & 16 \\
& Spastic unilateral & 7 \\
\multirow{2}{*}{ GMFCS level } & Dyskinetic & 7 \\
& 1 & 3 \\
& 2 & 8 \\
& 3 & 6 \\
MACS level & 4 & 7 \\
& 5 & 6 \\
& 1 & 0 \\
& 2 & 6 \\
& 3 & 7 \\
& 4 & 9 \\
\hline
\end{tabular}

CP, cerebral palsy; GMFCS, Gross Motor Function Classification System; MACS, the Manual Ability Classification System. 
ity domains were used. The PEDI-FSS measures what the child can do in her/his everyday environment (capability) and each question is scored as 0 , indicating "cannot do" and 1 , indicating "can do." The CAS catch what the child actually does in her/his everyday environment (performance). The scores depend upon the extent of the caregiver's help, with 0 indicating full dependence and 5 indicating independence.

The GMFM-66 is an abbreviated version of the GMFM, which is a standardized tool developed to measure the gross motor function in children with $\mathrm{CP}$ in a specific test situation, without the use of mobility aids or orthoses. The GMFM-66 consists of 88 items grouped into five dimensions of gross motor function including lying and rolling, sitting, crawling and kneeling, standing, and walking, running, and jumping.

\section{Statistical Analysis}

The SPSS 23 (SPSS Statistics for Windows, Version 23.0; IBM Corporation, Armonk, NY, USA) program was used for demographic data and correlation analysis. The pre and posttreatment differences were analyzed with the paired t-test. Correlations between the extracted ICF-CY code set and PEDI and the GMFM-66 subdomain scores were analyzed with Pearson's correlation coefficient $(r=0.10-0.29$ was weak, $r=0.30-0.49$ was moderate, and $r \geq 0.50$ was a strong correlation [11].

\section{RESULTS}

The study was completed with 30 (17 males and 13 females) children with $\mathrm{CP}$. The demographic characteristics such as $\mathrm{CP}$ classification, GMFCS level, and the Manual Ability Classification System (MACS) level are given in Table 1. The mean age of the children with CP was $5.4 \pm 4.4$ years. As shown in Table 1, 16 subjects had spastic bilateral type $\mathrm{CP}$, seven had the spastic unilateral type, and seven had the dyskinetic type. The GMFCS level of three subjects was 1 , eight subjects had a level of 2 , six subjects had a level of 3 , seven subjects had a level of 4 , and six subjects had a level of 5 . The MACS level of six subjects was 2 , seven subjects had a level of 3 , nine subjects had a level of 4 , and eight subjects had a level of 5 .

Tables 2-4 give a summary of the coding related to the ICF codes with clinical assessments. Most of the requested functions in the GMFM-66 were classified in the activity component of the ICF, but most of the PEDI functions were classified in both the activity and participation components of the ICF.
Table 2. Extracted ICF-CY codes linked to the GMFM-66

\begin{tabular}{ll}
\hline List of codes & \multicolumn{1}{c}{ Category names } \\
\hline$d 4100$ & Lying down \\
d4101 & Squatting \\
d4103 & Sitting \\
d4104 & Standing \\
d4105 & Bending \\
d4106 & Shifting the body's center of gravity \\
d4107 & Rolling over \\
d4150 & Maintaining a lying position \\
d4153 & Maintaining a sitting position \\
d4154 & Maintaining a standing position \\
d4155 & Maintaining head position \\
d4200 & Transferring oneself while sitting \\
d4351 & Kicking \\
d4500 & Walking short distances \\
$d 4502$ & Walking on different surfaces \\
$d 4550$ & Crawling \\
$d 4551$ & Climbing \\
$d 4552$ & Running \\
$d 4553$ & Jumping
\end{tabular}

ICF-CY, The International Classification of Functioning, Disability, and Health-Child and Youth version; GMFM-66, Gross Motor Function Measure-66.

The GMFM-66 coded 19 items in the mobility domain of the ICF. PEDI mobility and mobility assist were coded in 29 items and 13 items respectively in the mobility domain of the ICF. In addition, the self-care function of the PEDI was coded in 13 items and self-care assist was coded in 13 items.

The correlation between the extracted ICF-CY codes and the PEDI subdomain and the GMFM-66 was found to be strongly acceptable. There were strong negative correlations between the extracted ICF-CY codes and the PEDI scores $(r=0.76-0.95$; $\mathrm{p}<0.05$ ). There were strong positive correlations between the extracted ICF-CY codes and the GMFM-66 scores $(r=0.76-$ 0.95; p < 0.05) (Table 5).

\section{DISCUSSION}

The main findings in the present study revealed that the extracted ICF-CY codes showed a high correlation with the GMFM-66 and PEDI. Therefore, it can be useful in evaluating the functional conditions of patients with $\mathrm{CP}$ in relation to mobility and self-care in the pediatric rehabilitation area. To the best of our knowledge, no previous study investigated the validity of the ICF-CY codes extracted by clinical matching, making this study the first in this respect.

In our study, ICF-CY and clinical evaluation charts show a negative correlation. The reason is that ICF-CY means a lower 
Table 3. Extracted ICF-CY codes linked to the PEDI mobility function

\begin{tabular}{|c|c|c|c|}
\hline \multicolumn{2}{|r|}{ PEDI mobility function } & \multicolumn{2}{|r|}{ PEDI mobility assist } \\
\hline List of codes & Category names & List of codes & Category names \\
\hline $\mathrm{d} 4100$ & Lying down & $\mathrm{d} 4101$ & Squatting \\
\hline $\mathrm{d} 4101$ & Squatting & $d 4102$ & Kneeling \\
\hline$d 4102$ & Kneeling & $d 4103$ & Sitting \\
\hline$d 4103$ & Sitting & d4104 & Standing \\
\hline d4104 & Standing & d4105 & Bending \\
\hline d4105 & Bending & $\mathrm{d} 4106$ & Shifting the body's center of gravity \\
\hline $\mathrm{d} 4106$ & Shifting the body's center of gravity & $\mathrm{d} 4107$ & Rolling over \\
\hline $\mathrm{d} 4107$ & Rolling over & $\mathrm{d} 420$ & Transferring oneself while sitting \\
\hline d420 & Transferring oneself while sitting & $d 4551$ & Climbing \\
\hline $\mathrm{d} 430$ & Lifting & d4556 & Shuffling \\
\hline$d 4450$ & Pulling & $\mathrm{d} 4600$ & Moving around within the home \\
\hline$d 4451$ & Pushing & $d 4601$ & Moving around within buildings other than home \\
\hline$d 4452$ & Reaching & $\mathrm{d} 4602$ & Moving around outside the home and other buildings \\
\hline$d 4453$ & Turning or twisting the hands or arms & & \\
\hline d4454 & Throwing & & \\
\hline$d 4455$ & Catching & & \\
\hline d4500 & Walking short distances & & \\
\hline $\mathrm{d} 4502$ & Walking on different surfaces & & \\
\hline$d 4503$ & Walking around obstacles & & \\
\hline$d 4550$ & Crawling & & \\
\hline$d 4551$ & Climbing & & \\
\hline d4552 & Running & & \\
\hline d4553 & Jumping & & \\
\hline d4555 & Scooting and rolling & & \\
\hline d4556 & Shuffling & & \\
\hline d4600 & Moving around within the home & & \\
\hline $\mathrm{d} 4601$ & Moving around within buildings other than home & & \\
\hline$d 4602$ & Moving around outside the home and other buildings & & \\
\hline d465 & Moving around using equipment & & \\
\hline
\end{tabular}

ICF-CY, International Classification of Functioning, Disability, and Health-Child and Youth version; PEDI, Pediatric Evaluation of Disability Inventory.

Table 4. Extracted ICF-CY codes linked to the PEDI self-care function

\begin{tabular}{clll}
\hline & PEDI self-care function & & PEDI self-care assist \\
\hline List of codes & Category names & List of codes & Category names \\
\hline$d 510$ & Washing oneself & $d 510$ & Washing oneself \\
$d 5200$ & Caring for skin & $d 5200$ & Caring for skin \\
$d 5201$ & Caring for teeth & $d 5201$ & Caring for teeth \\
$d 5202$ & Caring for hair & $d 5202$ & Caring for hair \\
$d 5205$ & Caring for nose & $d 5205$ & Caring for nose \\
$d 5300$ & Regulating urination & $d 5300$ & Regulating urination \\
$d 5301$ & Regulating defecation & $d 5301$ & Regulating defecation \\
$d 5400$ & Putting on clothes & $d 5400$ & Putting on clothes \\
$d 5401$ & Taking off clothes & $d 5401$ & Taking off clothes \\
$d 5402$ & Putting on footwear & $d 5402$ & Putting on footwear \\
$d 5403$ & Taking off footwear & $d 5403$ & Taking off footwear \\
$d 550$ & Eating & $d 550$ & Eating \\
$d 560$ & Drinking & $d 560$ & Drinking \\
\hline
\end{tabular}

ICF-CY, International Classification of Functioning, Disability, and Health-Child and Youth version; PEDI, Pediatric Evaluation of Disability Inventory.

functional level as the score increases, but GMFM66 and PEDI mean a higher functional level as the score increases. In the extracted ICF-CY codes, the body structure components were not revealed as it was rarely covered by clinical measures just like the GMFM-66 and PEDI. The extracted GMFM-66 and PEDI content differed significantly in their representation of the ICF-CY codes. In the component of activity and participation, the GMFM only covered subdomain d4 (mobility) 


\begin{tabular}{|c|c|c|c|c|c|}
\hline & & \multicolumn{2}{|c|}{ Pre } & \multicolumn{2}{|c|}{ Post } \\
\hline & & $r$ & $\mathrm{p}$ & r & $\mathrm{p}$ \\
\hline \multirow[t]{3}{*}{ ICF-CY mobility } & GMFM-66 & 0.89 & 0.01 & -0.95 & 0.01 \\
\hline & PEDI mobility function & -0.92 & 0.01 & -0.95 & 0.01 \\
\hline & PEDI mobility assist & -0.76 & 0.01 & -0.83 & 0.01 \\
\hline \multirow[t]{2}{*}{ ICF-CY self-care } & PEDI self-care function & -0.95 & 0.01 & -0.93 & 0.01 \\
\hline & PEDI self-care assist & -0.93 & 0.01 & -0.80 & 0.01 \\
\hline
\end{tabular}

ICF-CY, International Classification of Functioning, Disability, and Health-Children and Youth version; GMFM-66, Gross Motor Function Measure-66; PEDI, Pediatric Evaluation of Disability Inventory.

compared to the PEDI, which included d4 (mobility) and $\mathrm{d} 5$ (self-care). Another study showed that PEDI included additional b1 (mental functions) and e1 (products and technology) subdomains [12]. We excluded the social functional area of the PEDI because it included too many items. The GMFM was highly recommended by health professionals as the first choice and the gold standard tool for assessing gross motor function in children with CP [13]. In our study, the GMFM-66 mapped to the components of the activity area. PEDI and GMFM-66 primarily cover only the components of activity and participation. These findings suggest a lack of good representation of the important interactions between the child's personal characteristics and the environment. However, our study provides novel information on how to characterize each measure based on the ICF language.

The selection of a proper outcome measure depends upon many factors including the psychometric properties and instrument's content, the type of intervention and its effect, and the availability of a version for the child's specific age [14]. In our study, the GMFM-66 and PEDI were highly associated with the extracted ICF-CY codes at the pre and post-treatment times. However, there was no significant correlation in the amount of change over time. This finding implies that ICF-CY code extraction is a meaningful evaluation tool limited to use as a cross-sectional score in the mobility and self-care areas.

The ICF-CY contains many areas that the GMFM and PEDI do not. However, such broadness makes the clinical application more difficult [15]. In addition, some ICF-CY categories were not specific enough to fully represent the unique content of some items of the GMFM and PEDI. But there are various benefits of the ICF-CY in the pediatric rehabilitation area. First, it contains domains that are important for describing functional mobility and its environments. Therefore, it can be applied across different parts of management such as assess- ment, goal-setting, and the outcome measures of intervention. Secondly, the ICF-CY may enhance communication among professionals, and among professionals and parents because it offers a common inter-professional language. Therefore, identifying the ICF domains related to the GMFM and PEDI is preparatory work necessary for the clinical application of the ICF-CY.

A limitation of our study was that the ICF codes extracted from the PEDI only included the mobility and self-care chapters. However, the PEDI included additional mental functions and products and technology subdomains and did not reflect the all domain of the PEDI. Additionally, the total sample size of our study was 30 . Therefore, a separate analysis by age groups such as preschool, school, and adolescents could not be done. Future studies should be performed with more samples including the separate analysis and clinical assessment of additional areas.

\section{CONCLUSIONS}

The present study revealed that the extracted ICF codes showed a high correlation with clinical assessments such as the GMFM-66 and PEDI. The extracted ICF codes were a valid tool for evaluating the mobility and self-care conditions of $\mathrm{CP}$ in the pediatric rehabilitation area. Further studies that consider more variables such as mental and environmental factors and social support for children and their families are needed.

\section{CONFLICTS OF INTEREST}

No potential conflict of interest relevant to this article was reported. 


\section{AUTHOR CONTRIBUTIONS}

Conceptualization: JSK, SDP, SHY. Data curation: SDP. Formal analysis: JSK, SDP. Investigation: JSK, SDP. Methodology: JSK. Project administration: SDP. Resources: JSK. Visualization: JSK. Writing - original draft: JSK. Writing - review \& editing: JSK, SDP, SHY.

\section{ORCID}

Sang-Duk Park, https://orcid.org/0000-0002-1510-5118

Sook-Hee Yi, https://orcid.org/0000-0003-2996-3284

\section{REFERENCES}

1. Lollar DJ, Simeonsson RJ. Diagnosis to function: classification for children and youths. J Dev Behav Pediatr 2005;26(4):32330.

2. World Health Organization. International classification of functioning, disability and health: children and youth version: ICF-CY. Geneva: World Health Organization; 2007.

3. Escorpizo R, Finger ME, Glässel A, Gradinger F, Lückenkemper M, Cieza A. A systematic review of functioning in vocational rehabilitation using the international classification of functioning, disability and health. J Occup Rehabil 2011; 21(2):134-46.

4. Sheehan D, Robertson L, Ormond T. Comparison of language used and patterns of communication in interprofessional and multidisciplinary teams. J Interprof Care 2007;21(1):17-30.

5. Haley SM. Pediatric evaluation of disability inventory (PEDI): development, standardization and administration manual. Boston: PEDI Research Group, New England Medical Center Hospital; 1992.

6. Palisano R, Rosenbaum P, Walter S, Russell D, Wood E, Galuppi B. Development and reliability of a system to classify gross motor function in children with cerebral palsy. Dev Med Child
Neurol 1997;39(4):214-23.

7. Smits DW, Ketelaar M, Gorter JW, van Schie P, Dallmeijer A, Jongmans M, et al. Development of daily activities in schoolage children with cerebral palsy. Res Dev Disabil 2011;32(1): 222-34.

8. Davis E, Waters E, Mackinnon A, Reddihough D, Graham HK, Mehmet-Radji 0, et al. Paediatric quality of life instruments: a review of the impact of the conceptual framework on outcomes. Dev Med Child Neurol 2006;48(4):311-8.

9. Ostensjø S, Carlberg EB, Vøllestad NK. Everyday functioning in young children with cerebral palsy: functional skills, caregiver assistance, and modifications of the environment. Dev Med Child Neurol 2003;45(9):603-12.

10. Cieza A, Geyh S, Chatterji S, Kostanjsek N, Ustün B, Stucki G. ICF linking rules: an update based on lessons learned. J Rehabil Med 2005;37(4):212-8.

11. Khetani MA, Cliff AB, Schelly C, Daunhauer L, Anaby D. Decisional support algorithm for collaborative care planning using the participation and environment measure for children and youth (PEM-CY): a mixed methods study. Phys Occup Ther Pediatr 2015;35(3):231-52.

12. Schiariti V, Klassen AF, Cieza A, Sauve K, O'Donnell M, Armstrong R, et al. Comparing contents of outcome measures in cerebral palsy using the international classification of functioning (ICF-CY): a systematic review. Eur J Paediatr Neurol 2014;18(1):1-12.

13. Vargus-Adams JN, Martin LK. Measuring what matters in cerebral palsy: a breadth of important domains and outcome measures. Arch Phys Med Rehabil 2009;90(12):2089-95.

14. Palisano RJ, Orlin M, Chiarello LA, Oeffinger D, Polansky M, Maggs J, et al. Determinants of intensity of participation in leisure and recreational activities by youth with cerebral palsy. Arch Phys Med Rehabil 2011;92(9):1468-76.

15. Bickenbach J, Cieza A, Rauch A, Stucki G. ICF core sets: manual for clinical practice. Göttingen: Hogrefe; 2012. 